OAI-PMH: http://www.indteca.com/ojs/index.php/Revista Scientific/oai

Ensayo Original / Original Essay

\title{
Transcomplejidad en la Educación Primaria: Investigación Transcompleja
}

\author{
Autor: César Enrique López Arrillaga \\ Universidad Latinoamericana y del Caribe, ULAC \\ prof.cesarlopez@gmail.com \\ Caracas, Venezuela \\ https://orcid.org/0000-0002-2926-8508
}

\section{Resumen}

El proceso de enseñanza y aprendizaje en las instituciones educativas de educación primaria se orienta desde la complejidad de las realidades actuales, en el marco de la transdisciplinariedad de los factores que fluyen en las interacciones de los actores educativos, el objetivo del presente ensayo, es realizar un abordaje teórico de la Transcomplejidad en la Educación Primaria, basado con los aportes de Sánchez (1995), y otros referentes teóricos como Villegas (2010), en función de establecer el análisis y vinculación desde el entorno de las escuelas que desarrollan procesos de formación en el nivel primaria, desde la Transcomplejidad orientada en la educación transcompleja, partiendo de las premisas que los estudiantes son diversos, únicos e irrepetibles en su personalidad, intereses y necesidades de formación bajo la mirada de la integralidad del hecho educativo en el contexto de la educación primaria.

Palabras clave: educación; escuela primaria; docente de escuela primaria; investigación.

Fecha de Recepción:

19-08-2019
Fecha de Aceptación:

30-11-2019
Fecha de Publicación: 05-02-2020 


\title{
Transcomplexity in Primary Education: Transcomplex Research
}

\begin{abstract}
The teaching and learning process in educational institutions of primary education is oriented from the complexity of current realities, within the framework of transdisciplinarity of the factors that flow in the interactions of educational actors, the objective of this essay is to perform a theoretical approach to Trans-Complexity in Primary Education, based on the contributions of Sánchez (1995), and other theoretical references such as Villegas (2010), based on establishing the analysis and linkage from the environment of schools that develop training processes at the primary level, from Trans-Complexity oriented to trans-complex education, based on the assumptions that students are diverse, unique and unrepeatable in their personality, interests and training needs under the gaze of the integrality of the educational fact in the context of primary education.
\end{abstract}

Keywords: education; primary school; primary school teacher; research.

How to cite this essay:

López, C. (2020). Transcomplexity in Primary Education: Transcomplex Research. Revista Scientific, 5(15), 305-319, e-ISSN: 2542-2987. Recovered from: https://doi.org/10.29394/Scientific.issn.2542-2987.2020.5.15.15.305-319

Date Received:

19-08-2019
Date Acceptance:

30-11-2019
Date Publication:

05-02-2020 


\section{Introducción}

En la actualidad la educación del Siglo XXI, se desarrolla desde el enfoque de múltiples, diversas y complejas realidades que convergen en la era de la globalización y postmodernidad de la humanidad, en cuanto hace referencia a fenómenos sociales y educativos en la nueva estructura de sociedad que emerge de las interacciones humanas a través de las nuevas tecnologías de la comunicación e información conjugadas con una educación actual que no responde a los procesos de aprendizajes y necesidades del estudiante de hoy.

Al respecto, se hace necesario comprender el proceso de enseñanza y aprendizaje en el contexto de educación primaria como transcomplejo, desde la praxis docente orientada a la investigación transcompleja en función de generar la construcción del conocimiento desde una cosmovisión integradora, holística, compleja, transdisciplinaria desde las realidades diversas, complejas, fluctuantes y dinámicas del estudiante relacionado con una educación transcompleja desde el paradigma de la complejidad.

En este orden, la transcomplejidad se presenta orientada por enfoques postmodernos que se relaciona con los procesos, procedimientos, actos y realidades en las organizaciones desde una perspectiva de transcomplejidad, de allí que el presente ensayo se basará en los referentes teóricos integrando los postulados del paradigma de la complejidad, además el análisis de los principios de la investigación transcompleja, la transcomplejidad en la educación primaria como proceso de valorar e interpretar la diversidad de los actores del hecho educativo desde su particularidades y personalidades únicas y propias que interactúan en la escuela.

De acuerdo con las consideraciones anteriores, con el objetivo de vislumbrar teóricamente la transcomplejidad en el contexto de las escuelas de educación primaria en el marco de una educación del Siglo XXI, planteado como la interpretación y valoración educativa como un todo alineado con el 
todo de los procesos que la integran para la formación de seres humanos con valores éticos y morales, tal como lo señala Morín (2003): "la parte está en el todo y el todo está en la parte" (pág. 68).

Es decir, relacionado con las realidades múltiples que se desarrollan de manera cotidiana en las escuelas de educación primaria, que, en la mayoría de los hechos, situaciones, eventos o fenómenos educativos son pocos comprendidos por los actores del proceso de enseñanza y aprendizaje, en la construcción de saberes y conocimientos, surgiendo las barreras para una educación que de respuestas a las exigencias de la sociedad actual desde su complejidad.

\section{Desarrollo}

\subsection{La Transcomplejidad en la Educación Primaria}

Al referirnos a la transcomplejidad en el contexto de la educación primaria, se hace necesario partir de la percepción de considerar el entorno escolar como espacio para la promoción, reconocimiento y valoración de las corrientes de pensamientos diversos para la reflexión sobre las realidades complejas de cada actor educativo, que se relacionan con la existencia de seres humanos en interrelaciones mutuas en una construcción continua e infinita de saberes, vivencias, y conocimientos para la transformación de su ambiente que lo rodea.

En ese mismo contexto, el proceso de enseñanza-aprendizaje se vincula estrechamente con la transcomplejidad en tal sentido, durante el desarrollo de las acciones, estrategias, actividades del hecho educativo, se evidencia en los actores participantes actitudes y realidades complejas reúnen un conjunto de percepciones cognitivas e interese de formación que permite la construcción colectiva desde la individualidad del conocimiento orientada a los intereses comunes de los participantes del proceso educativo desde la integración multidimensional y universal. Las afirmaciones anteriores sugieren 
que, desde las premisas de coordinar, guiar el proceso educativa orientadas a la acción y praxis del docente en el contexto de la educación primaria partiendo de su conocimiento individual relacionado con sus habilidades y destrezas en la actitud de como relacionarse con el mundo, enseñar, comunicar y analizar en base a la realidad compleja de los estudiantes en una formación transdiciplinaria que convergen las diversas áreas del conocimiento enmarcada en los valores para el forjamiento de ciudadanos holísticos y críticos que la sociedad desde su complejidad requiere para las transformación que exige el Siglo XXI, se relaciona con lo planteado por Woods (1998), en:

El manejo y el control del propio conocimiento y de la propia manera de conocer pueden contribuir a mejorar las maneras de cómo afrontar el mundo, tales como: anticipar, reflexionar, enseñar, comunicar, discriminar, resolver problemas, discutir, argumentar, confrontar los propios puntos de vistas con los otros, desarrollar el poder de discernimiento, etc. (pág. 87).

Dentro de este marco, la educación primaria se considera el nivel de formación en la etapa de desarrollo humano más importante del estudiante, desde la cosmovisión holística y continua en concordancia con la realidades complejas que surgen en la cotidianidad, de manera transversal, espontanea, natural y simultánea, es decir, se percibe un ambiente que se desarrolla en el devenir de la integralidad, espiritualidad, unicidad, diversidad desde la mirada de la transcomplejidad del ser y su entorno en las forma de comprender el mundo.

En ese mismo contexto, el docente en el contexto escolar del nivel de primaria se orienta en su práctica educativa del aula, desde la acción dialógica con los estudiantes, siendo así, un hecho educativo para el dialogo en función de construir el conocimiento colectivo basado en el intercambio de reflexiones para superar las barreras y la educación tradicional desde la constatación de ideas en la simplicidad a lo complejo para la formación relacionada con la complejidad a los ciudadanos con la finalidad de generar las transformaciones 
de la sociedad del Siglo XXI, tal como lo expresa, Sánchez (1995):

La aplicación del trabajo en el aula en función del análisis del discurso para construir conocimiento, llama a la reflexión para dejar de lado estereotipos educativos cerrados basados en evaluaciones por exámenes con contenidos rígidos, por procesos de confrontación de ideas, de diálogos significativos, vinculando conocimientos en todos los niveles, es decir desde el más simple al más complejo, pasando por capacidades comunicativas que lleven a su defensa situaciones de interacción social (pág. 165).

Al mismo tiempo, la capacidad de los actores educativos está vinculada con la habilidad, destreza y uso de la creatividad desde la inteligencia de transcender los criterios, currículo, pensum, planes, programas, paradigmas de la educación tradicional mecanicista, para impulsar una transformación del sistema educativo desde la comprensión de la complejidad de los procesos, por lo cual, amerita la puesta en práctica de acciones, estrategias y métodos de transcomplejidad, para consolidar una formación ser social desde su realidad compleja.

De este modo, emerge el proceso de enseñanza y aprendizaje transcomplejo, que se desarrolla con la interrelación de los estudiantes y docentes desde las realidades complejas existentes en el marco de la escuela de educación primaria, para consolidad una educación transcompleja que propicie la integración, interrelación y confrontación de ideas, saberes y vivencias en el hecho educativo transcomplejo, en el cual cada persona es protagónicas de su formación de acuerdo a su ritmo individual, igualmente, el docente es guía de los aprendizajes y garantiza el contrataste y discusión de los planteamientos en el aula, como se ilustra en la figura 1. 
Figura 1. Proceso de Enseñanza y Aprendizaje Transcomplejo.

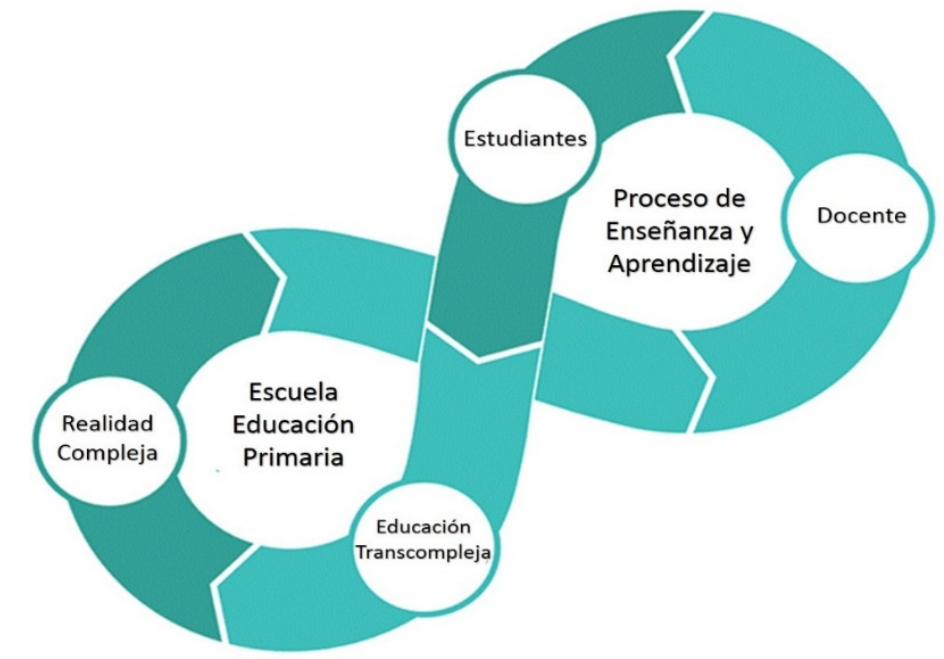

Fuente: El Autor (2019).

El Proceso de Enseñanza y Aprendizaje: se orienta en función de los pasos, actividades, estrategias, procedimientos, planes, programas, indicadores, objetivos, proyectos pedagógicos de aula (PPA), proyectos educativos integrales comunitarios (PEIC), orientaciones pedagógicas del Ministerio del poder popular para la Educación (MPPE) en conjunto con la comunidad educativa: Consejos Educativos, Consejos Comunales, Comunas y demás organizaciones del poder popular ubicadas en el contexto adyacente de la escuela, planteado desde una integralidad de todos los actores que confluyen en la corresponsabilidad de la formación de los estudiantes bajo la premisa de la holística y la complejidad de sus intereses, necesidades, habilidades y destrezas con el propósito de genera el ser social capaz de hacer las transformación que exige la sociedad actual. En relación con lo señalado por Pérez (2004): "la complejidad hace de la enseñanza un proceso constante inclusividad, basado en la investigación desde la interdisciplinariedad" (pág. 196). 
Dentro de este marco, los Estudiantes representan el propósito esencial del proceso educativo, es el centro en que convergen y se transversaliza todas las acciones y actuaciones educativas desarrolladas con las estrategias de aprendizajes que propicien la satisfacción de las necesidades, intereses desde la complejidad de la realidad social, en el marco de una práctica educativa transcompleja de múltiples y transdiciplinarias actitudes humanas, en la formación del estudiante como ciudadano bajo la mirada de la transcomplejidad orientada al humanismo, lo espiritual, consciente de un mundo planetario en sociedad del Siglo XXI.

En este mismo aspecto, los Docentes ejercer la tarea de guiar el proceso de enseñanza y aprendizaje, conscientes de la transcomplejidad educativa, relacionados con la aplicación de estrategias, acciones, proyectos y promover el aprendizaje desde la comprensión de los interés y necesidades del estudiante en su contexto transcomplejo. Es decir, una práctica educativa desde la complejidad de la realidad en el contexto de educación primaria, mediante la integración y la transversalización de todos los actores educativos en la formación compleja de los estudiantes como seres para la humanidad planetaria. Asimismo, en la formación del docente se fundamenta en la complejidad transcendiendo la formación tradicional.

Al respecto, la Educación Transcompleja se relaciona a promover acciones transdiciplinarias en los procesos educativos que se orienten en la integralidad, unicidad, holos, espiritualidad, necesidades, intereses de cada estudiante, valorando su etapa de desarrollo humanos en correlación con su nivel de estudio, mediante un desarrollo continuo en permanente adquisición mediante el dialogo surja la construcción del conocimiento en colectivo, forjando así, el ciudadano deseado por la sociedad actual, que responda a las exigencias de una comunidad planetaria, ecológica y espiritual resaltando los valores más preciados para resguarda la sobrevivencia de la humanidad en las próximas generaciones y el planeta como comunidad global. 
Este orden de ideas, la Escuela de Educación Primaria representa por excelencia el espacio ideal para las interrelaciones humanas desde la transcomplejidad de las realidades individuales que convergen en la cotidianidad educativa, de allí que, se hace necesario la interpretación conceptual la concepción de la escuela como entorno formatico transcomplejo de la interpelación, confrontación en un contraste de ideas, pensamientos, saberes, vivencias conocimiento a través del dialogo como eje transversal de la formación transdiciplinaria del ser en las escuelas de educación primaria.

Por lo tanto, la Realidad Compleja hace referencia a las características, costumbres, tradiciones, elementos históricos, relaciones interpersonales de un contexto determinado, partiendo de la diversidad de preceptos y visiones individuales que enriquecen desde la composición compleja de la realidad, es decir, en el contexto de la escuela primaria emerge una realidad compleja en el marco de la transversalidad de relaciones cotidianas, surgiendo fenómenos educativos en los actores que fluyen en las instituciones educativas. Tal como lo expresa, Morín (2001a): las "cualidades individuales más ricas emergen en el seno del sistema social" (pág. 131).

Lo anteriormente expuesto, devela un proceso de enseñanza y aprendizaje desde la transcomplejidad vinculado de la percepción del dialogo como medio de contrastar, reflexionar en el marco de la construcción colectiva del conocimiento, mediante la guía del docente el proceso de formación compleja del estudiantes, incluyendo la escuela como espacio de materialización de la realidad compleja que enriquece desde la vivencias cotidianas la educación en proceso de transición, bajo la mirada del proceso educativo transcomplejo. Cabe desatacar lo planteado por Briz (2003), al señalar que: "el reconocimiento de la necesidad imperativa de asumir la complejidad existente en la realidad es intrínsicamente compleja" (pág. 276). 


\subsection{Investigación Educativa Transcompleja}

Es importante plantearse que a través de la investigación surge conocimientos en el contexto de la educación, planteado desde la perspectiva del proceso educativo desde las premisas de lo humanista, aprendizajes significativos y lo considera como seres pertenecientes a una sociedad compleja que se confronta en una realidad transcompleja como la indagación y contratación del aprendizaje complejo de los actores educativos en el contexto de educación primaria. Para Schavino y Villegas (2010), la conceptualizan como:

Un proceso bio-afectivo cognitivo, pero también socio-culturalinstitucional-político de producción de conocimientos, como un producto complejo que se genera de la interacción del hombre con la realidad de la cual forma parte. Este enfoque supera las disyunciones sujeto-objeto, y abre camino a lo interaccional y a lo reticular, como fuentes constitutivas de la realidad compleja (pág. 4).

De eso se desprende, la relación que surge desde la realidad transcompleja desarrollada en las escuelas de educación primaria en interacción continua, permanente, cíclica del estudiantes integrado al proceso educativo, en la construcción de saberes y formación desde la complejidad, en función, de recorrer un sendero de interacciones en el marco de una constitución transversal de la realidad compleja, en consecuencia, el docente como guía del aprendizaje complejo es quien propicia a través de las estrategias la superación de las barreras que limitan en algunas ocasiones el éxito de la formación del ser social en las instituciones educativas del nivel de primaria, es importante, resaltar lo mencionado por Morín (2001b): "tendrían que enseñarse estrategias que permitan afrontar los riesgos, lo inesperado, lo incierto y modificar su desarrollo en virtud de las informaciones adquiridas en el camino" (pág. 17). 
De acuerdo con lo anterior, la investigación en el ámbito educativo se desarrolla con la inclusión de estrategias y elementos de fortalezcan el proceso de enseñanza y aprendizaje. Al respecto, es importante indicar la pertinencia de un enfoque transcomplejo en la investigación educativa desde el planteamiento de una visión de comprensión de la transcomplejidad que surge en la escuela de educación primaria. En consecuencia, se devela los elementos de la investigación educativa transcompleja para su desarrollo, como se ilustra en la figura 2 .

Figura 2. Elementos de la Investigación Educativa Transcompleja.

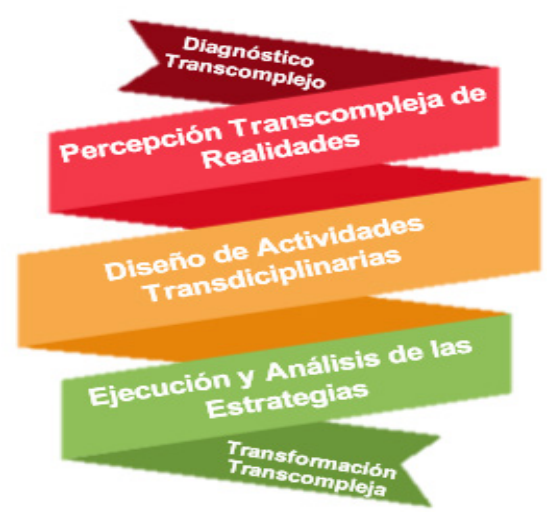

Fuente: El Autor (2019).

En el Diagnostico Transcomplejo se encuentra relacionado con la evaluación inicial del docente con el propósito de percibir a través de una observación integral y registro de las expresiones, manifestaciones que surjan del encuentro inicial con los estudiantes, con la finalidad. Seguidamente, la Percepción transcompleja de las realidades considerada como la forma de observar, ver y comprender las realidades diversas, transdiciplinarias, universales, únicas e irrepetibles en las interacciones de los actores educativos en el contexto de la educación primaria desde la concepción de la transcomplejidad educativa.

En este sentido, el Diseño de actividades transdiciplinarias se 
orientan en base de la realidad compleja en el proceso educativo, por lo que surge la necesidad de atender con estrategias que transciendan lo tradicional dando paso a lo creativo e innovador para la transformación del hecho educativo desde la dirección de una educación que responda a las exigencia del Siglo XXI, es decir, promover la transformación que exige la sociedad desde la práctica del docente como guía de la formación holística de los estudiantes a través de las tecnológicas y su relación con el contexto social.

Así mismo, la Ejecución y Análisis de las Estrategias vinculadas con el rol y papel del docente en el contexto educativo, el cual es ejercido como guía de la formación propiciado las más idóneas estrategias que corresponda con la realidad transcompleja que se desarrolla en la cotidianidad que emerge de las interacciones con los demás actores educativos en la escuela primaria, de allí que, se hace necesario el análisis y ejecución de las estrategias y el impacto en la creación del clonamientos de la individualidad en el marco de la colectividad de intercambios de saberes, vivencias y sentimientos en los estudiantes.

En consecuencia, la Transformación Transcompleja se deriva de la interrelación de los elementos del proceso educativo de la educación primaria en el marco de la investigación educativa transcompleja, como proceso sucesivo, dinámico, transdiciplinario que se fundamentado en la complejidad de los seres humanos que se vinculan en un intercambio mediante el dialogo, confrontación con el propósito de la construcción de conocimientos y saberes para consolidar el surgimiento del ser social capaz de promover las transformaciones en la sociedad actual, como ciudadanos críticos, participativos, democráticos y alineados a los altos valores humanos en el marco de una humanidad planetarias bajo la mirada ecológica y espiritual.

En líneas generales, a través del concurso y la transdisciplinariedad de los elementos descritos anteriormente, se devela una construcción de saberes, conocimientos, vivencias, experiencias, métodos y paradigma para una 
transformación transcompleja de la educación primaria en la formación de los ciudadanos desde una cosmovisión compleja de diversas realidades.

\section{Reflexiones finales}

La educación en el contexto de la educación primaria representa un nivel de importancia para la formación integral y transcompleja relacionada con los interés, necesidades y requerimientos en el marco de las realidades complejas de cada estudiante partiendo de la individualidad, unicidad, creatividad, integridad y los factores externos de la realidad social que convergen en sus actitudes y personalidad como personas que se transforman a través de las interrelaciones con los demás actores educativos en el hecho educativo como espacio ideal para la contratación, discusión y análisis de conocimientos desde la transdisciplinariedad de los saberes y aprendizajes.

Visto que, el docente desarrolla un papel como guía de la formación desde la transcomplejidad, desarrollando estrategias en consecuencia a las realidades del estudiante, para la consolidación de un proceso de enseñanza baso en transcender la visión educativa tradicional, dando paso la educación del Siglo XXI centrada en los seres humanos con los objetivos de un desarrollo universal desde la cosmovisión de una sociedad planetaria, ecológica y espiritual que preserve las generaciones futuras de la comunidad mundial.

Finalmente, la investigación el ámbito educativo impulsa la consolidación del conocimiento desde la transcomplejidad de los sucesos, eventos, acontecimientos, vivencias observadas en las interrelaciones complejas entre los actores educativos de la escuela de educación primaria, consolidando desde la curiosidad en el estudiante, para el impulso de indagar, dialogar y generar conocimientos pertinentes su formación como el ser social que requiere la sociedad actual. Finalmente, cabe señar la importancia de observar desde la transcomplejidad el contexto de educación primaria mediante la investigación compleja para fortalecer la formación de los 
ciudadanos y ciudadanas que asumirán las trasformaciones de la naciente sociedad planetaria.

\section{Referencias}

Briz, I. (2003). ¿Una realidad compleja? Apuntes y anotaciones para una teoría emergente en arqueología. RAMPAS. Revista AtlánticaMediterránea de Prehistoria y Arqueología Social, 6(1), 275-302, eISSN: 1138-9435 Recuperado de: https://dx.doi.org/10.25267/Rev atlmediterr prehist arqueol soc.2003.v6.07

Morín, E. (2001a). Introduction à la pensée complexe. París, Francia: Editions du Seuil.

Morín, E. (2001b). El Método I. La naturaleza de la naturaleza. Sexta edición. Madrid, España: Ediciones Cátedra.

Morín, E. (2003). Introducción al pensamiento complejo. España: Editorial Gedisa.

Pérez, E. (2004). Para pensar en la formación del docente venezolano del siglo XXI. Revista de Teoría y Didáctica de las Ciencias Sociales, (9), 189-208, e-ISSN: 1316-9505. Recuperado de:

https://www.redalyc.org/articulo.oa?id=65200911

Sánchez, T. (1995). La Construcción del aprendizaje en el aula. ISBN: 9505501374. Buenos Aires, Argentina: Magisterio del Rio de la Plata.

Schavino, N., \& Villegas, C. (2010). Espacio Iberoamericano del Conocimiento: De la teoría a la praxis en el enfoque integrador transcomplejo. Buenos Aires, Argentina: Congreso Iberoamericano de Educación "Metas 2021". Recuperado de:

http://www.adeepra.org.ar/congresos/Congreso\%20IBEROAMERICAN O/EIC/R0721 Schavino.pdf

Woods, P. (1998). Experiencias críticas en la enseñanza y aprendizaje. ISBN: 84-493-0456-3. Barcelona, España: Ediciones Paidós. 


\section{César Enrique López Arrillaga}

e-mail: prof.cesarlopez@gmail.com

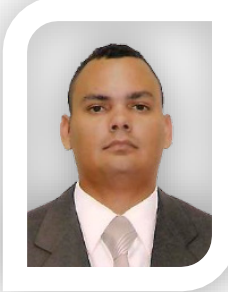

Nací en La Guaira, estado Vargas, Venezuela, el 7 de mayo del año 1985. Licenciado en Educación de la Universidad Nacional Experimental Simón Rodríguez (UNESR); Magister Scientiarum en Educación Superior de la Universidad Nacional Experimental de la Fuerza Armada (UNEFA); Comisionado Municipal de la Oficina Antidrogas del municipio Independencia; Director de Recursos Humanos de la Alcaldía del municipio Independencia; Jefe de Personal de la Policía Municipal Independencia; Director de Administración, Finanzas y Presupuesto de la Contraloría del municipio Simón Bolívar; Tutor de Trabajos Especiales de Grado (Especializaciones y Maestrías) en la Universidad Pedagógica Experimental Libertador (UPEL) y la UNEFA; Docente de Educación Media General en la Unidad Educativa Nacional 28 de Marzo; Coordinador de Control de Estudios de la Unidad Educativa Privada Coronel Antonio Nicolas Briceño; Docente de Educación Primaria de la Unidad Educativa Nacional Bolivariana Guaicaipuro. Arbitro Calificado de la Revista Scientific (e-ISSN: 2542-2987).

El contenido de este manuscrito se difunde bajo una Licencia de Creative Commons ReconocimientoNoComercial-Compartirlgual 4.0 Internacional 